\title{
Phonon sidebands of color centers in hexagonal boron nitride
}

\author{
P. Khatri, ${ }^{1}$ I. J. Luxmoore, ${ }^{1}$ and A. J. Ramsay ${ }^{2}$ \\ ${ }^{1}$ College of Engineering, Mathematics and Physical Sciences, University of Exeter, Exeter EX4 4QF, United Kingdom \\ ${ }^{2}$ Hitachi Cambridge Laboratory, Hitachi Europe Ltd., Cambridge CB3 OHE, United Kingdom
}

(Received 6 March 2019; revised manuscript received 24 July 2019; published 16 September 2019)

\begin{abstract}
Low temperature photoluminescence spectra of the phonon sidebands of a color center in hexagonal boron nitride are compared to an independent boson model. We infer that the LA-phonon sideband is described by deformation coupling proportional to in-plane strain, resulting in a phonon bath that is effectively twodimensional. For optical phonons, sharp resonances close to turning points in the phonon dispersion are observed. We infer that the TO-electron coupling is described by deformation coupling proportional to in-plane lattice displacement, resulting in a TO bath that is also effectively two-dimensional. By contrast, for the LO branches we infer that the Fröhlich coupling depends on the interactions between adjacent layers, and propose that the 200-meV peak is a signature of a few-layer sample. This work highlights features of electron-phonon coupling that arise from the layered structure of h-BN.
\end{abstract}

DOI: 10.1103/PhysRevB.100.125305

\section{INTRODUCTION}

The photon indistinguishability of a single-photon source based on a quantum dot or color center is degraded by phononassisted emission, which may be rejected at the expense of brightness using a wavelength filter, or improved through cavity enhancement of the zero-phonon line [1]. The electronphonon interactions also determine the fidelity with which the emitter can be prepared in the excited state [2,3]. In this respect, due to their large carrier wave functions, InAs quantum dots have the best optical coherence properties with lifetime limited dephasing, and about $96 \%$ emission into the zero-phonon line $[4,5]$. However, due to the large carrier wave functions, the cutoff energy of the electron-phonon interaction $\hbar \omega_{c}=1.3 \mathrm{meV}$ [6] restricts this to low temperatures, since $\hbar \omega_{c} / k_{B}=16 \mathrm{~K}$.

Recently, high temperature single-photon emission of color centers in hexagonal boron nitride (h-BN) have been reported [7,8] up to $800^{\circ} \mathrm{C}$ [9]. While there are reports of room temperature single photon emission in InGaN quantum dots $[10,11]$, color centers in diamond [12] and $\mathrm{SiC}$ [13], h-BN stands out for the high ZPL fraction of the emission. h-BN is a layered material with a graphenelike lattice. It has a large bandgap, and is used as an insulator in two-dimensional (2D) electronics. This raises the question of whether the highly anisotropic crystal structure leads to a phonon bath that is effectively three dimensions or two dimensions, and if so, does the reduced dimensionality have an advantage for the optical coherence properties of the emitter. The effects of dimensionality on acoustic-phonon-assisted emission of quantum dots in nanostructures has recently been investigated theoretically $[14,15]$. The issue has been experimentally investigated for quantum dots in carbon nanotubes with a onedimensional (1D) phonon bath [16]. Recently, Vuong et al. [17] have analyzed the acoustic phonon sidebands of UVemitting defect-bound excitons in bulk h-BN, and explain the results in terms of a three-dimensional (3D) acoustic phonon bath with an angle-averaged speed of sound. There are a couple of reports that fit the acoustic and optical sidebands at room [18] and low temperature [19].

Here we analyze the phonon sidebands of emission from the color center in multilayer flakes of h-BN in detail. By comparison of acoustic sidebands of PL data to an independent boson model, typically used for quantum dots [20], we infer the "order" of the electron-phonon spectral density $J(\omega) \sim \omega^{n} f^{2}(\omega)$ as, $n=2$, where $f(\omega)$ is a form factor. This arises due to deformation coupling of LA phonons to the in-plane $\mathrm{k}$ vector, due to the in-plane polarization of the LA phonons in h-BN [21], and a phonon dispersion that is nearly independent of $k_{z}$ for phonon energies larger than $10 \mathrm{meV}$. The optical phonon band has a number of resonances coinciding with turning points in the phonon dispersion. We propose that a peak at $200 \mathrm{meV}$, also reported by others [19] is a signature of the few-layer sample. The peaks associated with TO phonons are best described by a deformation coupling proportional to in-plane lattice displacement. Combined with the near degeneracy of the TO band with respect to out-ofplane momentum [22], the TO bath is also effectively twodimensional. By contrast, although the polarization vector of LO phonons is in-plane, we infer that the Fröhlich coupling depends on interactions between the layers. This may partly explain variations in the optical-phonon sidebands between different emitters. Compared to emitters in a 3D host material, this work identifies qualitative differences in the emitterphonon coupling that arise from the layered structure of h-BN. For a second emitter, evidence for coupling to out-of-plane polarized phonon modes is reported.

\section{PHOTOLUMINESCENCE MEASUREMENTS}

The sample consists of few-layer flakes of hBN (Graphene Supermarket) drop-cast onto a silicon substrate, which then undergoes a rapid thermal anneal in nitrogen atmosphere, 


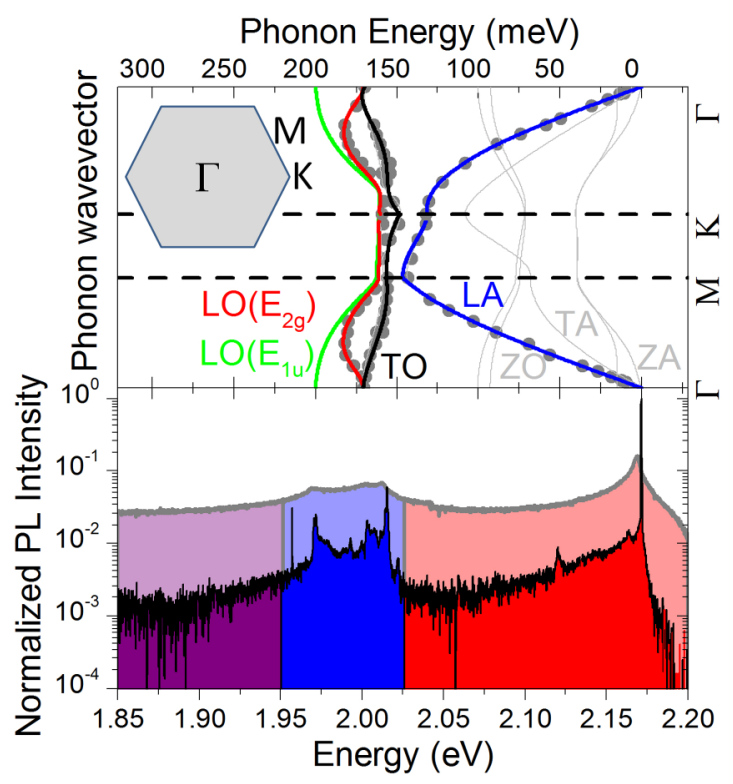

FIG. 1. Comparison of PL at $20 \mathrm{~K}$ and $275 \mathrm{~K}$ for emitter I. The ZPL can be identified by its large drop in intensity with temperature. By comparison to the phonon dispersion curves [21] the acoustic and optical sidebands can be identified. The markers indicate data points, and lines are fits to data from Ref. [21]. For the $\operatorname{LO}\left(\mathrm{E}_{1 \mathrm{u}}\right)$ band, a fit to $a b$ initio calculations in Ref. [21] is used. (Inset) 2D Brillouin zone.

ramping up to $850^{\circ} \mathrm{C}$ in $7 \mathrm{~min}$ and holding for $8 \mathrm{~min}$, before being allowed to cool. The studied samples are most likely multilayer flakes of a few $\mu \mathrm{m}^{2}$ area. For a 532-nm pump laser, the density of emitters is low, under 1 per $100 \mu \mathrm{m}^{2}$. We focus on two color centers emitting close to $2.17 \mathrm{eV}$.

The identity of the color center is unknown, and the subject of some debate. However, in Ref. [23], in monolayer hBN samples where the emitters are at approximately $580 \mathrm{~nm}$, the dominant defects in TEM were found to be the boron vacancy. Therefore, the defects studied here may be the $V_{B}^{-}$.

Figure 1 compares the micro-photoluminescence spectra of emitter I using a 532-nm pump at temperatures of 20 and $275 \mathrm{~K}$. Close to room temperature, the spectrum consists of a bright, narrow line at $2.171 \mathrm{eV}$. We attribute this peak to the zero phonon line (ZPL) emission of the color center, since the intensity of the peak is sensitive to temperature. The energy of the ZPL is $159 \mathrm{meV}$ less than the photon energy of the pump laser, similar to the energy of an optical phonon. Defects emitting at similar energies have been previously reported [24]. Although the setup is polarization dependent, both the emission and absorption are found to be approximately colinearly polarized, consistent with previous reports [25]. At $275 \mathrm{~K}$, there is a slight asymmetry of the ZPL, which is clearly revealed as the temperature is decreased. In Fig. 1(a), the spectra is color coded to indicate red-detuned sidebands due to acoustic phonon emission assisted radiative recombination (detuning $<150 \mathrm{meV}$ )(red), and optical phonon emission assisted radiative recombination $(150<$ detuning $<200 \mathrm{meV})$ (blue).

At low temperature, approximately $18 \%$ of the emitted photons are from the zero phonon line at $571 \mathrm{~nm}$. This is high compared to $12 \%, 3.7 \%$ at $575 \mathrm{~nm}$, and $682 \mathrm{~nm}$, reported in
Ref. [24], and considerably smaller than the $80 \%$ at $623 \mathrm{~nm}$ reported in Ref. [7]. The coupling of the color center to the vibronic modes, as evident in the temperature-dependent spectra of Fig. 1, leads to the observed reduction in the relative intensity of the ZPL as the temperature is increased. About $19 \%$ of the emission is into the optical phonon band, indicating that coupling to optical phonons is efficient, and the broad acoustic phonon band accounts for the rest.

\section{ANALYSIS OF ACOUSTIC PHONON SIDEBAND}

To analyze the phonon sidebands we compare the data to a model that considers a two-level system coupled to a bath of phonons. If the system is excited into the upper energy level at time zero, the electronic polarization, acting as the light source decays as

$$
P(t)=\exp \left(-\frac{\gamma_{\mathrm{ZPL}} t}{2}-\gamma(t)-i \Phi(t)\right)
$$

where $\gamma_{\mathrm{ZPL}}$ is a phenomenological dephasing rate to account for the finite width of the zero-phonon line. In the following, it is found from the FWHM of a Lorentzian fit to the ZPL. The phonon-induced dephasing is described by [26]

$$
\begin{aligned}
& \gamma(t)=\sum_{a} \int \frac{V_{d} d^{d} k}{(2 \pi)^{d}}\left|\frac{g_{k, a}}{\omega_{k, a}}\right|^{2}\left(1-\cos \omega_{k, a} t\right)\left(2 N\left(\omega_{k, a}, T\right)+1\right), \\
& \Phi(t)=\sum_{a} \int \frac{V_{d} d^{d} k}{(2 \pi)^{d}}\left|\frac{g_{k, a}}{\omega_{k, a}}\right|^{2} \sin \omega_{k, a} t .
\end{aligned}
$$

$g_{k, a}=g_{k, a}^{(1)}-g_{k, a}^{(0)}$ gives the difference in electron-phonon coupling between the upper (1) and lower (0) energy levels of the two-level system with a phonon of wave vector $k$ in band $a$, with energy $\hbar \omega_{k, a} . N(\omega, T)$ is the Bose-Einstein distribution. The PL spectrum is then calculated as $S(\omega)=$ $\operatorname{real}\left(\int_{0}^{\infty} d t e^{-i \omega t} P(t)\right)$. In the following, bulk phonon modes are assumed. The sum over $k$ is expressed as an integral over a $d$-dimensional k space of volume $V_{d}$. The energy dispersion is found from a fit to data in Ref. [21]. To interpolate between the $\Gamma-K$ and $\Gamma-M$ directions we approximate the dispersion in terms of the magnitude $k$ and direction $\theta$ of the $\mathrm{k}$ vector as

$$
\begin{aligned}
\omega^{2}(k, \theta) \approx & \frac{1}{2}\left(\omega_{\Gamma-K}^{2}(k)+\omega_{\Gamma-M}^{2}(k)\right) \\
& +\frac{1}{2}\left(\omega_{\Gamma-K}^{2}(k)-\omega_{\Gamma-M}^{2}(k)\right) \cos 6 \theta .
\end{aligned}
$$

To describe the electron-phonon coupling, we expand the electron-phonon coupling in terms of wave vector $k$, and consider the first two terms.

$$
g_{k, a}^{(b)}=\frac{1}{\sqrt{2 \rho_{d} V_{d} \hbar \omega_{k, a}}}\left(i \cdot \mathcal{M}_{a}^{(b)}+\mathbf{k} \cdot \hat{u}_{a} \mathcal{D}_{a}^{(b)}\right) f_{a}^{(b)}(k) .
$$

The prefactor normalizes the energy of the phonon mode to $\hbar \omega_{k, a} . \rho_{d}$ is the $d$-dimensional mass density. $f_{a}^{(b)}(k)$ is a form factor and is the overlap of the electron density of electronic state (b) and the phonon wave function $(k, a)$. For traveling waves, it is the Fourier transform of the electron probability density, and $f_{a}^{(b)}(0)=1$ by definition. The $\mathcal{M}$ term describes a piezolike change in the energy proportional to the lattice displacement, and the $\mathcal{D}$ term a deformationlike coupling proportional to the strain. For acoustic phonons, the $\mathcal{D}$ term 
dominates since the strain changes the separation between neighboring lattice sites. The unit vector $\hat{u}_{a}$ is the polarization of the phonon mode of band $a$.

In this work, our goal is to learn about the electron-phonon coupling by comparison of data to the above model. We reduce the number of fitting parameters, by noting that it is the difference in electron-phonon coupling $g_{k, a}$ that appears in Eq. (1). We therefore drop the index (b), and define $\mathcal{M}_{a}=$ $\mathcal{M}_{a}^{(1)}-\mathcal{M}_{a}^{(0)}$, and $\mathcal{D}_{a}=\mathcal{D}_{a}^{(1)}-\mathcal{D}_{a}^{(0)}$. We also approximate $g_{k, a}$ with a single form factor $f_{a}(k)$, defined such that $f_{a}(0)=$ 1. In the unlikely case that $\mathcal{M}_{a}^{1}=\mathcal{M}_{a}^{(0)}$, the form factor and coefficient are redefined such that $f(k \rightarrow 0)=k^{2}$, since the probability density is real. In the case of a quantum dot, the envelope of the carrier wave function spans more than $10^{3}$ lattice constants and is relatively unaffected by lattice vibrations. The electron-phonon coupling arises from a change in bandgap and hence is a property of the host material [20]. For a deep defect, the intraband transition is insensitive to changes in the bandgap. However, since the carrier envelope is similar in size to a lattice constant, the confinement potential is strongly influenced by lattice vibrations, and hence a coupling constant that is a property of the defect is expected.

To help identify the dimension and coupling mechanism of the acoustic phonons, we consider the spectral density $J(\omega)$ of the electron-phonon interaction, defined as

$$
\sum_{a} \int \frac{V_{d} d^{d} k\left|g_{k, a}\right|^{2}}{(2 \pi)^{d}} \rightarrow \int d \omega J(\omega)
$$

in Eq. (1). The spectral density more directly expresses the phonon sidebands, and to first order the red sideband is $S(\omega) \propto \frac{J(\omega)}{\omega^{2}}(1+N(\omega, T))$. In the low $k$ regime, where the dispersion is linear $J(\omega)=\alpha \omega^{n} f^{2}(\omega)$, where $n=d-1+$ $2 c-1+4 p$ describes the "order" of the coupling. For piezoand deformationlike coupling, $c=0,1$, respectively. For an upper and lower energy level with different electron-phonon coupling constants, $p=0, f(0)=1$ and if the coupling constants are the same, $p=1, f^{2}(k \rightarrow 0)=k^{4}$. The "order" determines the qualitative shape of the phonon sideband; $n=1$ is often referred to as the "Ohmic" case [15].

In Fig. 2, a closeup of the acoustic phonon sideband is shown. The exponential decrease of the phonon sideband with red-detuning implies an exponential form factor, $f^{2}(k)=$ $e^{-k \sigma}$, where $\sigma$ is a measure of the size of the electron density. The absence of resonances near $75 \mathrm{meV}$ and $100 \mathrm{meV}$, implies that the $\mathrm{ZO}$ phonons do not play a significant role. To identify the order of the electron-phonon interaction, a set of calculations assuming deformation coupling $(c=1)$, and that $\mathcal{D}^{(1)} \neq$ $\mathcal{D}^{(0)}, p=0$, and $n=d$ are made for longitudinal acoustic phonon baths with linear dispersion of different dimension $n=d=1,2,3$, and are shown alongside the data in Fig. 2(a). Only $d=n=2$ can describe the data. In principle, the case $p=1, c=0$, and $d=0$, could also explain the data, but this seems unlikely. Therefore the acoustic sideband arises from deformation coupling to a phonon bath that is effectively twodimensional. Since the form factor restricts the contributing phonons to low $\mathrm{k}$, it is difficult to distinguish between contributions from TA and LA phonons. An additional calculation using Eq. (3) to approximate the anisotropy and include the nonlinearity of the dispersion is also made (see Fig. 2) and

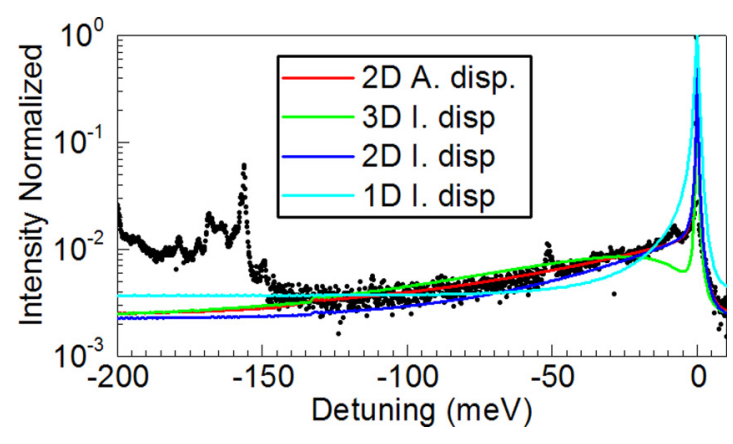

FIG. 2. Closeup of acoustic phonon sideband. Note that sideband decays exponentially with detuning. Lines are calculations that assume electron phonon spectral densities, $J(\omega) \sim \omega^{n} f^{2}(\omega)$ of order $n=1$ (cyan), 2 (blue), 3 (green). More specifically, the case $n=d$ where a deformation coupling with $f(0)=1$ is assumed. These calculations use a linear dispersion. Qualitatively, the $n=2$ case fits best. The red line shows the case $n=2$, using Eq. (3) to estimate the anisotropy of the nonlinear dispersion curves, and gives a slightly better fit to data. The parameters used are as follows: $\mathcal{D}_{1 D}=4 \mathrm{eV}, \rho_{1 D}=1.65 \times 10^{-16} \mathrm{~kg} \mathrm{~m}^{-1}, \sigma_{1 D}=$ $0.21 \mathrm{~nm} ; \quad \mathcal{D}_{2 D}=10.5 \mathrm{eV}, \rho_{2 D}=0.76 \mathrm{mg} \mathrm{m}^{-2}, \sigma_{2 D}=0.35 \mathrm{~nm}$; $\mathcal{D}_{3 D}=26 \mathrm{eV}, \rho_{3 D}=2.18 \times 10^{3} \mathrm{~kg} \mathrm{~m}^{-3}, \sigma_{3 D}=0.49 \mathrm{~nm}$.

provides a slightly better description of the data at higher phonon energy.

An effective two dimensional coupling can arise if the integrand of Eq. (1) is independent of out-of-plane k vector $k_{z}$. Since the polarization $\hat{u}_{a}$ of the LA and TA phonon modes is in-plane, the deformation coupling is to in-plane momentum, $g_{k, \mathrm{LA}} \sim \mathcal{D}_{\mathrm{LA}} \mathbf{k} . \hat{u}_{\mathrm{LA}} \rightarrow \mathcal{D}_{\mathrm{LA}} k_{\perp}$. In addition, for phonon energies $\hbar \omega_{k}>10 \mathrm{meV}$ the dispersion curves of the LA and TA phonons are also independent of $k_{z}$ [21]. Hence, due to the in-plane polarization of the phonon modes, for most energies of interest the electron-phonon coupling is effectively two-dimensional, although this is a multilayer sample.

If we assume that only LA phonons contribute, since k. $\hat{u}_{T A}=0$, we extract a value $\mathcal{D}_{2 D} \approx 10.5 \mathrm{eV}$, using $\rho_{2 D}=$ $0.76 \mathrm{mg} \mathrm{m}^{-2}$ [27]. This is reasonable, since deformation coupling is usually much stronger for LA than TA phonons. $\sigma_{\mathrm{LA}}=0.35 \mathrm{~nm}$ which is about 2.4 times the nearest neighbor separation of $0.144 \mathrm{~nm}$ [28].

The deformation coupling strength is close to the $\mathcal{D}_{\mathrm{LA}}=$ $11 \mathrm{eV}$ found for UV-emitting defect-bound excitons coupled to a 3D phonon bath in bulk h-BN [17], and is close to values found for excitons in GaAs QDs [2]. However, it is relatively large compared to deformation coupling constants of up to $0.6 \mathrm{eV}$ measured under static strain conditions for $\mathrm{hBN}$ color centers emitting at $2.14 \mathrm{eV}$ [29]. We also note that the deformation constant $\mathcal{D}_{\mathrm{LA}}$ needed to reproduce the data increases with dimension, implying that the electron-phonon interaction is enhanced for lower dimension systems.

\section{ANALYSIS OF OPTICAL PHONON SIDEBAND}

To gain insight into the interaction of the color center with optical phonons, we compare the dispersion curve of hBN measured in Ref. [21] to a closeup of the optical phonon sideband of the emission spectra taken at $20 \mathrm{~K}$; see Fig. 3. The red and green curves indicate bulk LO-phonon branches 


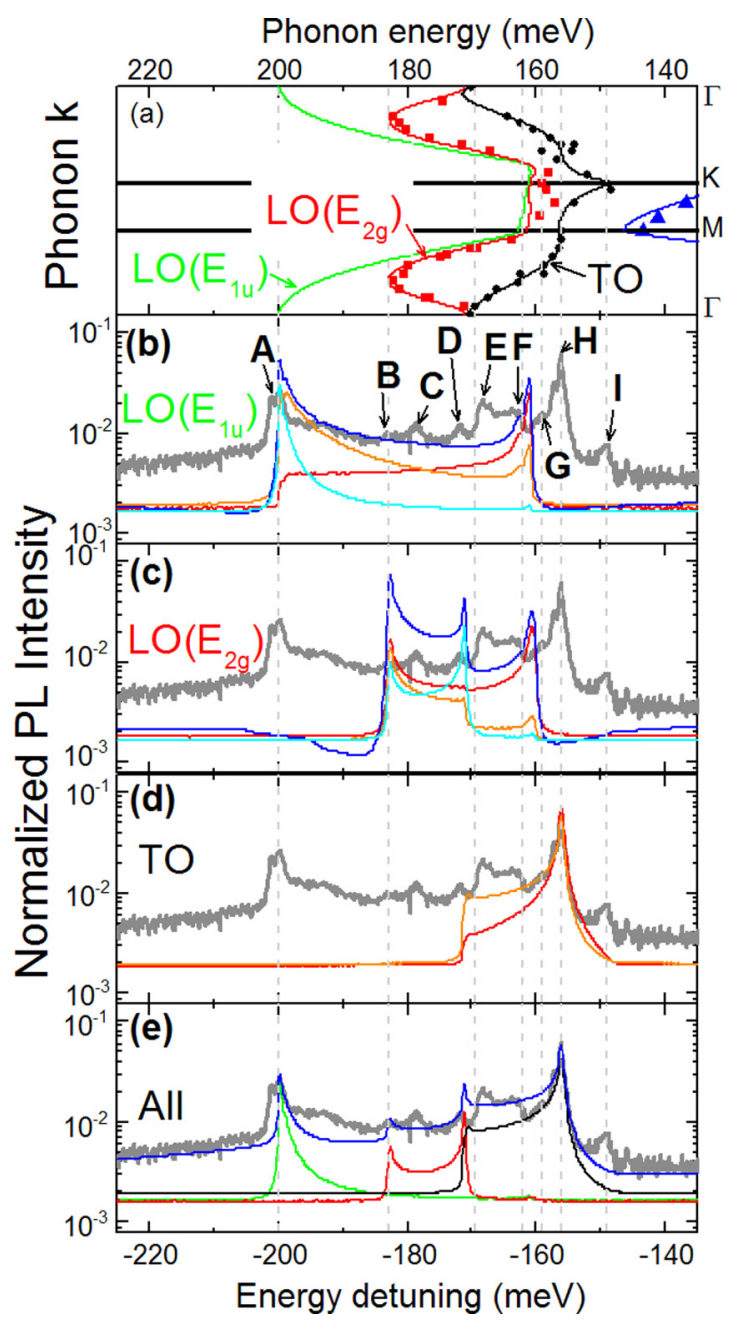

FIG. 3. (a) Dispersion curves of the optical phonon branches. The markers are data, and the lines are fits to data taken from Serrano et al. [21]. The $\operatorname{LO}\left(E_{1 u}\right)$ band is a fit to ab initio calculation of Ref. [21]. Vertical lines are aligned to turning points in the dispersion curves where the density of states is high. Comparison of data to calculations considering a single band: (b) $\operatorname{LO}\left(E_{1 u}\right)$, (c) $\mathrm{LO}\left(E_{2 g}\right)$, (d) TO. We consider a Fröhlich (blue, cyan) and deformation (red, orange) coupling with (blue, $\epsilon_{\mathrm{eff}}=22.2, \sigma=0$ ); (cyan, $\epsilon_{\mathrm{eff}}=33.3, \sigma=0.2 \mathrm{~nm}$ ); $\left(\mathrm{red}, \mathcal{M}=72 \mathrm{eV} \mathrm{nm}^{-1}\right.$ ); (orange, $\left.\mathcal{M}=341.5 \mathrm{eV} \mathrm{nm}^{-1}\right)$. For the $\mathrm{TO}$ branch only the deformation coupling is presented. (e) A fit to data assuming Fröhlich coupling to two LO branches, and deformation coupling to the TO. Values of $\epsilon_{\mathrm{eff}}\left(\mathrm{LO}\left(E_{1 u}\right)\right)=33.3, \epsilon_{\mathrm{eff}}\left(\mathrm{LO}\left(E_{2 g}\right)\right)=66.7, \sigma_{\mathrm{LO}}=0.2 \mathrm{~nm}, \mathcal{M}_{\mathrm{TO}}=$ $153 \mathrm{eV} \mathrm{nm}^{-1}, \sigma_{\mathrm{TO}}=0.1 \mathrm{~nm}$ are used.

with $E_{2 g}$ and $E_{1 u}$ symmetry at the $\Gamma$ point, where neighboring planes oscillate in-phase or antiphase, respectively. The black curve shows the bulk TO branch. The overbending of the in-phase $\mathrm{LO}\left(E_{2 g}\right)$ band results from a spring constant that changes sign with the separation between lattice sites; see Table I of Ref. [30]. For multilayers [22], adjacent out-ofphase layers $\left(k_{z} \neq 0\right)$ generate an in-plane electric field via the Coulomb interaction increasing the spring constants. This increases the energy of the $\operatorname{LO}\left(E_{1 u}\right)$ with respect to the $\operatorname{LO}\left(E_{2 g}\right)$ band, suppresses the overbending, and results in energies that depend on the number of layers, as seen for example in
TABLE I. List of optical phonon peaks observed in Fig. 3. Most peaks can be linked to turning points in the dispersion. $T$ labels a turning point away from the first Brillouin zone edge.

\begin{tabular}{lcc}
\hline \hline Peak & Detuning $(\mathrm{meV})$ & Identity \\
\hline $\mathrm{A}$ & 200 & $\mathrm{LO}\left(E_{1 u}, \Gamma\right)$ \\
$\mathrm{B}$ & 183 & $\mathrm{LO}\left(E_{2 g}, T\right)$ \\
$\mathrm{C}$ & 178 & $?$ \\
$\mathrm{D}$ & 172 & $\mathrm{LO}\left(E_{2 g}, \Gamma\right)$ \\
$\mathrm{E}$ & 169 & $\mathrm{TO}(\Gamma)$ \\
$\mathrm{F}$ & 163 & $\mathrm{LO}\left(E_{1 u}, M-K\right)$ \\
$\mathrm{G}$ & 159 & $\mathrm{LO}\left(E_{2 g}, M-K\right)$ \\
$\mathrm{H}$ & 156 & $\mathrm{TO}(\mathrm{M}), \mathrm{TO}(\mathrm{T})$ \\
$\mathrm{I}$ & 149 & $\mathrm{TO}(K)$ \\
\hline \hline
\end{tabular}

other 2D materials such as graphene [31], $\mathrm{MoS}_{2}$, and $\mathrm{WS}_{2}$ $[32,33]$. Several peaks can be observed. To aid identification, construction lines are drawn from the turning points in the bulk dispersion curves, corresponding to energies where the phonon density of states is high.

Table I compares the features labeled A-I with turning points in the dispersion curves [21] of the optical sidebands, where the phonon density of states are high. The strong peak-A at $200 \mathrm{meV}$, also seen in [19] can be unambiguously identified as the $\operatorname{LO}\left(E_{1 u}\right)$ band at the $\Gamma$ point of bulk h-BN [22]. If h-BN behaves similarly to other 2D materials, then this suggests that the defect resides in a multilayer with $\mathcal{N} \geqslant 4$ [31-33].

The lowest energy peak (I) corresponds to $\mathrm{TO}(\mathrm{K})$. The strongest peak $(\mathrm{H})$ at $156 \mathrm{meV}$ corresponds to the TO branch near to the first Brillouin zone edge. The relative strength of this peak compared to the LO peak (A) is suggestive of a sample with few layers, rather than a single layer, as the relative DOS of the TO branch compared with the LO branches is higher since the degeneracy of the TO branches is not lifted by the Coulomb interaction [22]. The presence of peak E suggests the turn-on of the electron-TO interaction at low momentum as the phonon energy decreases. Peak D is assigned to $\operatorname{LO}\left(E_{2 g}, \Gamma\right)$ assuming the energy is slightly higher than the $\mathrm{TO}(\Gamma)$ point. It is also reproduced by the calculations; see Fig. 3(c). Peaks F and G are near the Brillouin edge of the LO bands, and we use the energy ordering to assign F to $\operatorname{LO}\left(E_{1 u}, M-K\right)$ and $\mathrm{G}$ to $\operatorname{LO}\left(E_{2 g}, M-K\right)$. Peak B corresponds to the energy of the turning point in $\operatorname{LO}\left(E_{2 g}, T\right)$.

To model the electron-phonon interaction, we consider a Fröhlich-like coupling to the LO branch of dimension $d=2$ given by [34]

$$
\left|g_{k, \alpha, \mathrm{LO}}\right|^{2}=\frac{e^{2} \omega_{k, \alpha, \mathrm{LO}}}{2^{4-d} \hbar \epsilon_{0} V_{d} k_{\perp}^{d-1} \epsilon_{\mathrm{eff}, \alpha}} f_{\mathrm{LO}}^{2}(k),
$$

where $\epsilon_{\text {eff }}$ is treated as a fitting parameter. For a single conduction band electron,

$$
\frac{1}{\epsilon_{\mathrm{eff}}}=\frac{1}{\epsilon_{\infty}}-\frac{1}{\epsilon_{s}}
$$

where $\epsilon_{\infty}$ and $\epsilon_{s}$ are the high frequency and static dielectric constants, respectively. The other parameters are the permittivity of free space $\epsilon_{0}$, and the electron charge $e$. The 
anisotropy of the dispersion of the optical phonons is approximated according to Eq. (3). Note that qualitatively, there is no difference in $\gamma(t)$ for a 2D or 3D Fröhlich interaction, and we cannot use the data to distinguish between them. The polarization of the LO branch is in-plane, hence the argument in Eq. (6) is $k \rightarrow k_{\perp}$. For a $\mathcal{N}$-layer sample [22], there are $\mathcal{N}$ LO branches, labeled by the index $\alpha$ according to the relative phase between adjacent layers (i.e., $k_{z}$ ). The dispersion depends on the index $\alpha$, and the number of layers $\mathcal{N}$. In Fig. 3(a), the $\mathrm{LO}$ branches with $k_{z} c=0, \pi$ (i.e., $\operatorname{LO}\left(E_{2 g}\right), \operatorname{LO}\left(E_{1 u}\right)$, respectively) are shown for a bulk material. The volume $V_{d} \rightarrow$ $\mathcal{N} V_{2}$, and a sum over $\mathcal{N}$ branches is made in Eq. (1).

In addition to the Fröhlich interaction, it is expected that both the LO and TO modes will exhibit a coupling proportional to the optical displacement. For optical phonons, this is often referred to as deformation coupling [35], but can be treated as the $\mathcal{M}$ term in Eq. (2).

Figure 3 compares calculations of the different band contributions to the spectrum. The contribution to $\gamma(t)$ of both the Fröhlich interaction and the optical deformation coupling depends mostly on the inverse group velocity of the band. In Fig. 3(d), the TO branch is unaffected by additional layers [22], so should be less open to interpretation. The strongest peak $(\mathrm{H})$ is reproduced with $\mathcal{M}_{\mathrm{TO}}=72-341 \mathrm{eV} \mathrm{nm}^{-1}$, depending on the $\sigma_{\mathrm{TO}}$ used. This appears high, however, the origin of the coupling is the deformation of the lattice, and at the Brillouin edge the "acoustic" deformation coupling required to give the same shift in energy is $\mathcal{D}=\mathcal{M}_{\mathrm{TO}} a / \pi=$ 3.3-15.6 eV, which is not unreasonable. Peak I is not reproduced; this is attributed to the higher group velocity of the approximate dispersion curve used in our calculations.

In Fig. 3(b), it is clear that the 200-meV peak can only be explained by Fröhlich interaction to a bulklike $\operatorname{LO}\left(E_{1 u}\right)$ mode, since the $\mathcal{M}$ term has no peak at the $\Gamma$ point where $k_{\perp}=0$. Naively, one would expect the defect to couple to all of the LO modes equally. In Fig. 3(c), the $\mathrm{LO}\left(E_{2 g}\right)$ band has a higher DOS since the spread in energies is lower. Hence if the coupling were equal, this would imply that the peak at the $\mathrm{LO}(\mathrm{T})$ point would be stronger than the $200-\mathrm{meV}$ peak. This implies that for this defect, the Fröhlich coupling is stronger for LO modes where adjacent layers oscillate outof-phase. This may suggest that dipole fields generated by adjacent layers cancel or enhance the $\mathrm{E}$ field generated by the LO phonon, or may relate to which layer the defect resides. The strength of the interaction given by $\epsilon_{\text {eff }}^{-1}=0.03$ is small compared with the value of 0.056 for a single electron in bulk $\mathrm{h}-\mathrm{BN}$ [36]. This is to be expected since it is the difference in the charge distribution of the energy levels of optical transition that matters.

Figure 3(e) presents a fit to the optical sideband using two LO bands with Fröhlich coupling and a TO band with deformation coupling. We note that the model is overspecified, and the numbers used should not be considered as accurate. The discrepancy at high energy may suggest that more layers need to be considered, or that $\sigma_{\mathrm{LO}}<0.2 \mathrm{~nm}$.

\section{EMITTER II}

There is considerable variation in the phonon sidebands of the color centers. To make this point, Fig. 4 presents the

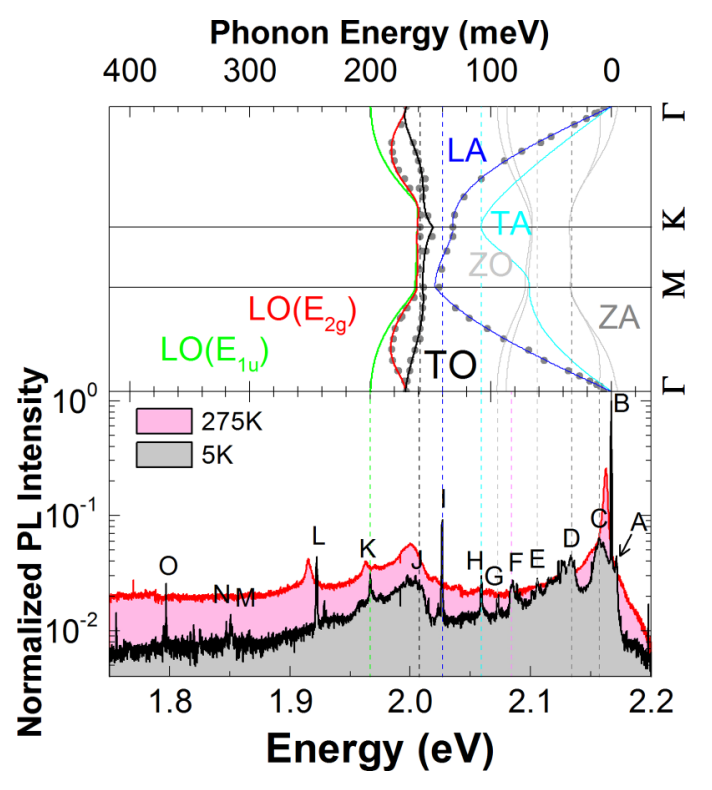

FIG. 4. Photoluminescence spectra of emitter II. (Top) Dispersion curves of bulk-phonon modes. The markers are data points, and the lines fit to data or $a b$ initio calculations of Ref. [21]. The z-polarized ZA and ZO branches are shifted to the blue by $5.5 \mathrm{meV}$ to match peak A. The peaks A-O are identified in Table II. To aid comparison a number of construction lines aligned with peaks in PL have been drawn.

photoluminescence spectrum of emitter II. Although the emission energies are similar, the spectrum is more complicated than for emitter I. Peak B is the strongest, and the peak intensity falls strongly with temperature. Hence we assign peak B as a ZPL. In Fig. 1, the ZPL is the highest energy peak, consistent with our two-level model. However, for emitter II the highest photon energy peak is the weaker peak A. We cautiously suggest that peak A is also a ZPL, either indicating a fine structure or a second color center. If we assign peaks $\mathrm{A}$ and $\mathrm{B}$ to $\mathrm{ZPLs}$, then peaks $\mathrm{C}-\mathrm{G}$ match turning points in the $\mathrm{ZA}$ and $\mathrm{ZO}$ dispersion curves relative to peak $\mathrm{A}$. The energies of peaks $\mathrm{C}$ and $\mathrm{G}$ also match expected turning points relative to peak $\mathrm{B}$. The energies of peaks $\mathrm{H}-\mathrm{O}$ match turning points of in-plane polarized phonon modes. The suggested identities of the peaks are presented in Table II. The out-of-plane polarized phonon modes have $B_{1 g}$ symmetry [21]. Observation of ZAand ZO-phonon-assisted emission suggests that one of the electron states of $\mathrm{ZPL}_{A}$ couples to this symmetry, possibly indicating a $p_{z}$ orbital [37]. We note that peak I is especially strong, sharp, and sensitive to temperature. This could indicate a ZPL at $610 \mathrm{~nm}$, or the $\mathrm{LA}(M)$ point with respect to peak B. Peaks H $[\mathrm{TA}(K)]$ and $\mathrm{L}[\mathrm{LA}(M)+\mathrm{TA}(K)]$ are prominent and correspond to displacements along the bond direction of the heavier nitrogen sublattice. Once again, there is a $200-\mathrm{meV}$ peak of the $\operatorname{LO}\left(E_{1 u}, \Gamma\right)$ point, indicating the sample has a few layers. Otherwise the in-plane optical phonon sideband has fewer features than emitter I, possibly due to broadening with ZA phonons, or the emitter preferentially couples to $\mathrm{TO}$ along the K direction where the DOS is less peaked. The energies of peaks M-O, match two phonon resonances given in Table II.

This suggests that either emitters I and II are from different species of defect, despite their similar emission energies 2.171 
TABLE II. List of peaks identified in Fig. 4 for emitter II. Peaks $A$ and $B$ are identified with ZPL lines labeled $z$ and $x$. The other peaks are identified relative to these two peaks. The bar labels modes where layers oscillate in antiphase. For peaks C and G the energy matches phonon energies with respect to both peaks $\mathrm{A}$ and $\mathrm{B}$.

\begin{tabular}{lcc}
\hline \hline Peak & Detuning $(\mathrm{meV})$ & Identity \\
\hline $\mathrm{A}$ & -5.5 & $\mathrm{ZPL}_{z}$ \\
$\mathrm{~B}$ & 0 & $\mathrm{ZPL}_{x}$ \\
$\mathrm{C}$ & 9.8 & $\mathrm{ZA}(\Gamma)_{z}$ or ZA $\left(B_{1 g}, \Gamma\right)_{x}$ \\
$\mathrm{D}$ & 32.7 & $\mathrm{ZA}(M, K)_{z}$ \\
$\mathrm{E}$ & 61 & $\mathrm{TA}(M)_{z} ?$ \\
$\mathrm{~F}$ & 83.2 & $\mathrm{ZO}(\Gamma)_{z}$ \\
$\mathrm{G}$ & 94 & $\mathrm{ZO}(\Gamma)_{z}$ or ZO $(\Gamma)_{x}$ \\
$\mathrm{H}$ & 107.6 & $\mathrm{TA}(K)_{x}$ \\
$\mathrm{I}$ & 140 & $\mathrm{LA}(M)_{x}$ \\
$\mathrm{~J}$ & 159 & $\mathrm{TO}(M)_{x}$ \\
$\mathrm{~K}$ & 200 & $\mathrm{LO}\left(E_{1 u}, \Gamma\right)_{x}$ \\
$\mathrm{~L}$ & 244.5 & $2 \mathrm{TO}(M)_{x}, 2 \mathrm{TA}(K)_{x}(M, K)_{x}$ \\
$\mathrm{M}$ & $315.5 \approx 2(158)$ & $\mathrm{TO}(K)_{x}+\left[\mathrm{LO}\left(E_{2 g}, \Gamma\right), \mathrm{TO}(\Gamma)\right]_{x}$ \\
$\mathrm{~N}$ & $319.8 \approx 149+170$ & $\mathrm{LO}\left(E_{1 u}, \Gamma\right)_{x}+\mathrm{TO}(\Gamma)_{x}$ \\
$\mathrm{O}$ & $369.4 \approx 200+170$ & \\
\hline \hline
\end{tabular}

vs $(2.167$ or 2.171$) \mathrm{eV}$, or the local environment of emitter II somehow activates the out-of-plane transition.

\section{CONCLUSIONS}

The phonon sidebands of a color center in h-BN emitting close to $2.17 \mathrm{eV}$ has been analyzed using an independent boson model usually applied to quantum dots [20]. Key differences in the electron-phonon coupling that arise due to the layered structure of h-BN are identified as follows. For emitter I, the acoustic sideband can be described by deformation coupling to an effective two-dimensional phonon bath with exponential form factor. This arises because the LA phonons are polarized in-plane, and deformation couple to in-plane momentum, and for phonon energies larger than $10 \mathrm{meV}$, the acoustic phonon dispersion is degenerate with respect to out-of-plane momentum. For a two-dimensional system, this results in an intrinsic sub-Lorentzian broadening of the ZPL [15], and could limit the optimum photon indistinguishability that could be achieved. However, looking at the dispersion curves, one might expect a crossover to an effective 3D phonon bath at low phonon energies. The identification of the order of the electron-phonon spectral density is important, since this determines a number of coherence properties, such as the power laws of intensity damping [2], and ZPL broadening [24,38-41].

The optical phonon band is significant. A peak at $200 \mathrm{meV}$, which can be attributed to Fröhlich coupling to an $\operatorname{LO}\left(E_{1 u}\right)$ phonon where neighboring layers vibrate in antiphase is prominent in both emitters, and is reported in Ref. [19]. We propose that this is a signature of a multilayer flake with $\mathcal{N}>3$. The LO phonons are Fröhlich coupled. From the data, we infer that contrary to our simple model, the Fröhlich interaction strength depends on the relative phase between adjacent layers, i.e., $k_{z}$. Furthermore, much of the variation in the optical phonon sideband between different emitters, may be down to the number of layers in the sample. The TO-electron coupling can be described by a deformation coupling proportional to the in-plane lattice displacement. Combined with the near degeneracy of the TO band with respect to the out-of-plane $\mathrm{k}$ vector, the TO bath is effectively two-dimensional.

For emitter II, we argue that a second weak ZPL coupled to $z$-polarized phonons is present.

We note that the cutoff length is small $\sigma_{\mathrm{LA}} \approx 0.4 \mathrm{~nm}$, and indicates small carrier wave functions. Due to the high speed of sound, this corresponds to a cutoff temperature of $\hbar \omega_{c} / k_{B}=\frac{\hbar v}{\sigma k_{B}} \approx 400 \mathrm{~K}$, where $v$ is the angle-averaged speed of sound, explaining the temperature robustness of the ZPL.

For bulk-phonon modes, an exponential form factor implies a Lorentizan-like [42] electron density in real space. The size of the electron density $\sigma$ varies between phonon branches. Within the independent boson model, this may arise from the use of a single form factor, and a different ratio of the coupling strength to the upper and lower energy levels of the optical transition between bands. However, this may also hint that short-lived phonon modes localized to the defect may be involved. How these should be treated in this model is an open question.

Following submission of this work, a few related publications have appeared [43-47].

\section{ACKNOWLEDGMENTS}

We gratefully acknowledge financial support from the Engineering and Physical Sciences Research Council UK under Grants No. EP/S001557/1 and No. EP/P026656/1. We thank J. A. Haigh for critical reading of the manuscript.
[1] J. Iles-Smith, D. P. S. McCutcheon, A. Nazir, and J. Mork, Nat. Photon. 11, 521 (2017).

[2] A. J. Ramsay, A. V. Gopal, E. M. Gauger, A. Nazir, B. W. Lovett, A. M. Fox, and M. S. Skolnick, Phys. Rev. Lett. 104, 017402 (2010).

[3] J. H. Quilter, A. J. Brash, F. Liu, M. Glassl, A. M. Barth, V. M. Axt, A. J. Ramsay, M. S. Skolnick, and A. M. Fox, Phys. Rev. Lett. 114, 137401 (2015).

[4] P. Borri, W. Langbein, S. Schneider, U. Woggon, R. L. Sellin, D. Ouyang, and D. Bimberg, Phys. Rev. Lett. 87, 157401 (2001).
[5] P. Borri, W. Langbein, U. Woggon, V. Stavarache, D. Reuter, and A. D. Wieck, Phys. Rev. B 71, 115328 (2005).

[6] A. J. Ramsay, T. M. Godden, S. J. Boyle, E. M. Gauger, A. Nazir, B. W. Lovett, A. M. Fox, and M. S. Skolnick, Phys. Rev. Lett. 105, 177402 (2010).

[7] T. T. Tran, K. Bray, M. J. Ford, M. Toth, and I. Aharonovich, Nat. Nanotechol. 11, 37 (2015)

[8] L. J. Martinez, T. Pelini, V. Waselowski, J. R. Maze, B. Gil, G. Cassabois, and V. Jacques, Phys. Rev. B 94, 121405(R) (2016). 
[9] M. Kianinia, B. Regan, S. Abdulkader Tawfik, T. T. Tran, M. J. Ford, I. Aharonovich, and M. Toth, ACS Photon. 4, 768 (2017).

[10] M. J. Holmes, K. Choi, S. Kako, M. Arita, and Y. Arakawa, Nano Lett. 14, 982 (2014).

[11] M. J. Holmes, S. Kako, K. Choi, M. Arita, and Y. Arakawa, ACS Photon. 3, 543 (2016).

[12] N. Mizuochi, T. Makino, H. Kato, D. Takeuchi, M. Ogura, H. Okushi, M. Nothaft, P. Neumann, A. Gali, F. Jelezko, J. Wrachtrup, and S. Yamasaki, Nat. Photon. 6, 299 (2012).

[13] A. Lohrmann, N. Iwamoto, Z. Bodrog, S. Castelletto, T. Ohshima, T. J. Karle, A. Gali, S. Prawer, J. C. McCallum, and B. C. Johnson, Nat. Commun. 6, 7833 (2015).

[14] P. Tighineanu, C. L. Dreessen, C. Flindt, P. Lodahl, and A. S. Sorensen, Phys. Rev. Lett. 120, 257401 (2018).

[15] G. M. Palma, K.-A. Suominen, and A. K. Ekert, Proc. R. Soc. A 452, 567 (1996).

[16] C. Galland, A. Hogele, H. E. Tureci, and A. Imamoglu, Phys. Rev. Lett. 101, 067402 (2008).

[17] T. Q. P. Vuong, G. Cassabois, P. Valvin, A. Ouerghi, Y. Chassagneux, C. Voisin, and B. Gil, Phys. Rev. Lett. 117, 097402 (2016).

[18] A. L. Exarhos, D. A. Hopper, R. R. Grote, A. Alkauskas, and L. C. Bassett, ACS Nano 11, 3328 (2017).

[19] M. A. Feldman, A. Puretzky, L. Lindsay, E. Tucker, D. P. Briggs, P. G. Evans, R. F. Haglund, and B. J. Lawrie, Phys. Rev. B 99, 020101(R) (2019).

[20] B. Krummheuer, V. M. Axt, and T. Kuhn, Phys. Rev. B 65, 195313 (2002).

[21] J. Serrano, A. Bosak, R. Arenal, M. Krisch, K. Watanabe, T. Taniguchi, H. Kanda, A. Rubio, and L. Wirtz, Phys. Rev. Lett. 98, 095503 (2007).

[22] K. H. Michel and B. Verberck, Phys. Rev. B 83, 115328 (2011).

[23] J. Feng, H. Deschout, S. Caneva, S. Hofmann, I. Loncaric, P. Lazić, and A. Radenovic, Nano Lett. 18, 1739 (2018).

[24] N. R. Jungwirth, B. Calderon, Y. Ji, M. G. Spencer, M. E. Flattel, and G. D. Fuchs, Nano Lett. 16, 6052 (2016).

[25] N. R. Jungwirth and G. D. Fuchs, Phys. Rev. Lett. 119, 057401 (2017).

[26] A. Vagov, V. M. Axt, and T. Kuhn, Phys. Rev. B 66, 165312 (2002).

[27] The mass density is estimated using a nearest neighbor lattice constant of $0.144 \mathrm{~nm}$ [28].

[28] R. Geick, C. H. Perry, and G. Rupprecht, Phys. Rev. 146, 543 (1966).
[29] G. Grosso, H. Moon, B. Lienhard, S. Ali, D. K. Efetov, M. M. Furchi, P. Jarillo-Herrero, M. J. Ford, I. Aharonovich, and D. Englund, Nat. Commun. 8, 705 (2017).

[30] K. H. Michel and B. Verberck, Phys. Rev. B 80, 224301 (2009).

[31] A. C. Ferrari, J. C. Meyer, V. Scardaci, C. Casiraghi, M. Lazzeri, F. Mauri, S. Piscanec, D. Jiang, K. S. Novoselov, S. Roth, and A. K. Geim, Phys. Rev. Lett. 97, 187401 (2006).

[32] A. Molina-Sanchez and L. Wirtz, Phys. Rev. B 84, 155413 (2011).

[33] C. Lee, H. Yan, L. E. Brus, T. F. Heinz, J. Hone, and S. Ryu, ACS Nano 4, 2695 (2010).

[34] F. M. Peeters, Wu Xiaoguang, and J. T. Devreese, Phys. Rev. B 33, 3926 (1986).

[35] X. B. Zhang, T. Taliercio, S. Kolliakos, and P. Lefebvre, J. Phys.: Condens. Matter 13, 7053 (2001).

[36] Ioffe semiconductors database, http://www.ioffe.ru/SVA/NSM/ Semicond/.

[37] M. Abdi, J.-P. Chou, A. Gali, and M. B. Plenio, ACS Photon. 5, 1967 (2018).

[38] B. Sontheimer, M. Braun, N. Nikolay, N. Sadzak, I. Aharonovich, and O. Benson, Phys. Rev. B 96, 121202(R) (2017).

[39] E. Neu, C. Hepp, M. Hauschild, S. Gsell, M. Fischer, H. Sternschulte, D. Steinmuller-Nethl, M. Schreck, and C. Becher, New J. Phys. 15, 043005 (2013).

[40] T. Muller, I. Aharonovich, Z. Wang, X. Yuan, S. Castelletto, S. Prawer, and M. Atature, Phys. Rev. B 86, 195210 (2012).

[41] K. D. Jahnke, A. Sipahigil, J. M. Binder, M. W. Doherty, M. Metsch, L. J. Rogers, N. B. Manson, M. D. Lukin, and F. Jelezko, New J. Phys. 17, 043011 (2015).

[42] In one dimension the Fourier transform of an exponential is a Lorentzian. In two dimensions it is a bit different.

[43] A. Bommer and C. Becher, Nanophotonics, 0123 (2019).

[44] D. Wigger, R. Schmidt, O. Del Pozo-Zamudio, J. A. Preuss, P. Tonndorf, R. Schneider, P. Steeger, J. Kern, Y. Khodaei, J. Sperling, S. M. de Vasconcellos, R. Bratschitsch, and T. Kuhn, 2D Materials 6, 035006 (2019).

[45] F. Paleari, H. P. C. Miranda, A. Molina-Sánchez, and L. Wirtz, Phys. Rev. Lett. 122, 187401 (2019).

[46] A. Dietrich, M. Burk, E. S. Steiger, L. Antoniuk, T. T. Tran, M. Nguyen, I. Aharonovich, F. Jelezko, and A. Kubanek, Phys. Rev. B 98, 081414(R) (2018).

[47] E. Cannuccia, B. Monserrat, and C. Attaccalite, Phys. Rev. B 99, 081109(R) (2019). 